\title{
Die Konstitution der Zeolithe.
}

\author{
Von \\ F. W. Clarke. ${ }^{1}$
}

Schon längst ist die augenscheinliche Verwandtschaft mancher Zeolithe mit den Feldspaten als wahrscheinlicher Schlüssel zu ihrer Konstitution erkannt worden, jedoch ist meines Wissens noch keine systematische Theorie über den Zusammenhang der beiden Gruppen aufgestellt. Einzelangaben liegen thatsächlich vor, wie die von StRH:NG in seiner Untersuchung über Chabazit, sowie von Fresensuts in seiner Auslegung von Phillipsit und Harmotom, indes fehlt bis jetzt eine allgemeine, zusammenfassende Erklärung aller Zeolithe. Doch ist zur Lösung dieser Aufgabe reichliches Material vorhanden, wie es sich ableitet aus der Entwässerung der Mineralien, der Art ihrer Zersetzung beim Schmelzen, ihrer synthetischen Bildung, ihren Änderungen unter dem Einflul's von Reagentien etc., und es würde das Endziel jeder erschöpfenden Diskussion über ihre Natur sein, alle diese Beweisstücke, alle diese verschiedenen Daten in Übereinstimmung zu bringen. Ohne Erfüllung dieser Bedingung kann kein System von konstitutionellen Formeln gültig sein; zugleich ist dann aber auch die Möglichkeit gegeben, dals beim Suchen nach zu grolser Allgemeinheit eine deutliche Harmonie erzwungen wird, wo wirkliche Beziehungen gar nicht existieren. Zur Vermeidung dieser Gefahr scheint es nötig, von vornherein anzunehmen, dal's nicht alle Zeolithe aus Feldspat entstanden sind; einige derselben sind Verbindungen von einem ganz verschiedenen Typus, doch lassen sich die meisten Glieder der Gruppe leicht durch eine allgemeine Ausdrucksweise darstellen, welche ebensogut für die Feldspate palst.

Die in vorliegender Abhandlung entwickelte Theorie der Zerlithe ist ein direkter Ausbau und eine Folge der Ansichten, welche ich seit einigen Jahren über die Glimmer, die Chlorite und verschiedene andere Mineralien veröffentlicht habe, und basiert auf den gleichen Prinzipien. Die komplexen, oder scheinbar komplexen Aluminiumsilikate werden allgemein als Substitutionsderivate einfacher normaler Salze, wie $\mathrm{Al}_{4}\left(\mathrm{SiO}_{4}\right)_{3}, \mathrm{Al}_{4}\left(\mathrm{Si}_{3} \mathrm{O}_{8}\right)_{3}$ etc. betrachtet, eine Vor-

1 Ins Deutsehe übertragen von Hermann Moraht. 
stellung, welche manche wichtigen Beziehungen zur Anschauung bringt und von allen Gesichtspunkten aus völlig gerechtfertigt erscheint. Ferner werden die Säureradikale $\mathrm{SiO}_{4}$ und $\mathrm{Si}_{3} \mathrm{O}_{8}$ als gegenseitig, das eine durch das andere, ersetzbar gedacht, welche Annahme jetzt durch die Arbeiten von Tschfrmaz über die Feldspathund Skapolith-Gruppe und durch meine Untersuchung über die Glimmergruppe mit Recht als gut begründet angesehen werden darf. Diese beiden fundamentalen Voraussetzungen bilden die Grundsteine, auf welchen weiter zu bauen ich versucht habe.

Die oben skizzierte Substitutionstheorie wurde schon in einer Abhandlung , ,über die chemische Struktur der natürlichen Silikate " auf die Feldspate angewandt, obwohl die Formeln nicht voll ausgeschrieben wurden. Die Natriumverbindung Albit $\mathrm{AlNaSi}_{3} \mathrm{O}_{8}$ ward als erstes Substitutionsderivat des unbekannten Salzes $\mathrm{Al}_{4}\left(\mathrm{Si}_{3} \mathrm{O}_{8}\right)_{3}$ und Anorthit als das entsprechende calciumbaltige Orthosilikat betrachtet. Verdreifacht man in beiden Fällen die einfachste Formel, so erhält man beifolgende Ausdrücke für die beiden Feldspatsalze:

$$
\begin{aligned}
\mathrm{Si}_{3} \mathrm{O}_{8} & =\mathrm{Na}_{3} \\
\mathrm{Al}-\mathrm{Si}_{8} \mathrm{O}_{8} & =\mathrm{Al} \\
\mathrm{Si}_{3} \mathrm{O}_{8} & =\mathrm{Al}
\end{aligned}
$$

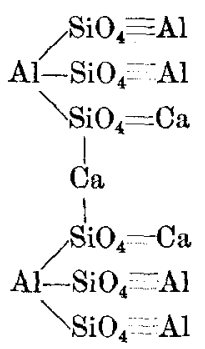

Unter der Annahme, dafs das Natriumsalz durch die entsprechende Orthoverbindung, sowie das Calciumsalz durch das analoge Trisilikat ersetzt werden kann, lassen sich aus obigen Formeln die Formeln mancher Zeolithe leicht ableiten. Während das Natriumorthosalz mit Nephelin isomer sein würde, ist die Calciumverbindung mit $\mathrm{Si}_{3} \mathrm{O}_{8}$ rein hypothetisch. Betrachten wir vorübergehend alles Wasser in den Zeolithen als Krystallisationswasser und bezeichnen die Gruppen $\mathrm{SiO}_{4}$ und $\mathrm{Si}_{3} \mathrm{O}_{8}$ durch das allgemeine Symbol X, so fällt nach obiger Grundlage die gröfsere Zahl der in Betracht kommenden Mineralien leicht unter zwei Gruppen mit folgenden allgemeinen Formeln:

1 Bull. U. S. Geolog. Survey (1887-1888) 60. 


$$
\begin{aligned}
& \text { 1. }\left\{\begin{array}{l}
\mathrm{Al}_{6} \mathrm{X}_{6} \stackrel{\mathrm{R}}{6}_{6}, \text { naq. }+ \\
\mathrm{AJ}_{3} \mathrm{X}_{3} \mathrm{R}_{3}, \text { naq. }
\end{array}\right. \\
& \text { 2. }\left\{\begin{array}{l}
\mathrm{Al}_{4} \mathrm{X}_{6} \mathrm{I}_{12}, \text { naq. }+ \\
\mathrm{Al}_{2} \mathrm{X}_{3} \stackrel{\mathrm{I}}{6}_{6}, \text { naq. }
\end{array}\right.
\end{aligned}
$$

Zur ersten Gruppe mit wechselnden Wassermengen gehört 'Thomsonit, Gismondit, Edingtonit, Phillipsit, Harmotom, Chabazit, Levynit, Gmelinit, Hydronephelith und Offretit, während der zweiten Gruppe Foresit, Laumontit, Heulandit, Epistilbit, Brewsterit, Skolezit, Stilbit, Faujasit und Natrolith angehören. In dieser Form verbleiben die beiden Gruppen indes nur, so lange wir das Wasser unberücksichtigt lassen, denn so wie wir das letztere teilweise als Konstitutionswasser betrachten, so müssen mehrere Glieder der ersten Reihe in die zweite versetzt werden. Das wird später noch deutlicher hervortreten. Die oben angeführten Arten sollen nun im besonderen in folgender Weise betrachtet werden:

Thomsonit. - $\mathrm{Al}_{6}\left(\mathrm{SiO}_{4}\right)_{6} \mathrm{Ca}_{3} \cdot 7 \mathrm{H}_{2} \mathrm{O}$. Derselbe entspricht dem Anorthit plus Wasser und liefert beim Schmelzen nach DoELter Anorthit. Gewöhnlich ist das Calcium teilweise durch Natrium ersetzt, wobei ein oder zwei Atome in dieser Weise ersetzbar sind. Bisweilen tritt, wie im „Mesole", ein Überschufs an Kieselsäure auf, welcher sich durch geringe Beimengungen von Trisilikatmolekülen oder $\mathrm{Si}_{3} \mathrm{O}_{8}$-Gruppen erklärt. Nach den Entwässerungsversuchen von DaMour, Hersch u. a. müssen $4 / 7$ des Wassers als Konstitutionswasser betrachtet werden, was sich darstellen lälst durch Ersetzen von $\mathrm{Al}$ durch $\mathrm{Al}=(\mathrm{OH})_{2}+\mathrm{H}_{2}$. Demnach wäre die endgültige Formel zu schreiben:

$$
\mathrm{Al}_{4}\left(\mathrm{SiO}_{4}\right)_{6} \mathrm{Ca}_{3}\left(\mathrm{AlH}_{2} \mathrm{O}_{2}\right)_{2} \mathrm{H}_{4} \cdot 3 \mathrm{H}_{2} \mathrm{O} \text {. }
$$

Gismondit. - Unsicher, jedoch wahrseheinlich $\mathrm{Al}_{6}\left(\mathrm{SiO}_{4}\right)_{6} \mathrm{Ca}$. $12 \mathrm{H}_{2} \mathrm{O}$, worin ein Teil des Calciums durch Kalium und vielleicht bisweilen $\mathrm{SiO}_{4}$ durch $\mathrm{Si}_{3} \mathrm{O}_{8}$ ersetzt ist. Bei $100^{\circ}$ geht angeblich $1 / 3$ des Wassers fort; sind die übrigen 8 Moleküle Konstitutionswasser, so würden vier Aluminiumatome durch $4\left(\mathrm{AlH}_{2} \mathrm{O}_{2}\right)+\mathrm{H}_{8}$ ersetzt sein und die Formel ergeben

$$
\mathrm{Al}_{2}\left(\mathrm{SiO}_{4}\right)_{6} \mathrm{Ca}_{3}\left(\mathrm{AlH}_{2} \mathrm{O}_{2}\right)_{4} \mathrm{H}_{8} .4 \mathrm{H}_{2} \mathrm{O} \text {. }
$$

Edingtonit. - Wahrscheinlich $\mathrm{Al}_{6} \mathrm{X}_{6} \mathrm{Ba}_{3} \cdot 12 \mathrm{H}_{2} \mathrm{O}$, wo $\mathrm{X} z \mathrm{zu}$ etwa $3 / 4$ aus $\mathrm{SiO}_{4}$ und $1 / 4$ aus $\mathrm{Si}_{3} \mathrm{O}_{8}$ besteht. Baryum ist teilweise durch andere Basen ersetzbar; die Art der Wässerung ist un- 
bestimmt. Nach Lemberg läfst sich Edingtonit oder eine ihm analoge Verbindung künstlich aus Natrolith erhalten. Obwohl Edingtonit die gleiche Gesamtzahl Wassermoleküle wie Gismondit besitzt, ist seine Krystallform eine gänzlich verschiedene, so dafs für beide Arten das Verhältnis von Krystall- zu Konstitutionswasser wahrscheinlich nicht das gleiche ist.

Phillipsit. - $\mathrm{Al}_{6} \mathrm{X}_{6} \mathrm{CaR}_{4} \cdot 15 \mathrm{H}_{2} \mathrm{O} . \stackrel{\mathrm{R}}{\mathrm{R}}$ kann $\mathrm{Na}$ oder $\mathrm{K}$ sein; das Verhältnis zwischen $\mathrm{SiO}_{4}$ und $\mathrm{Si}_{3} \mathrm{O}_{8}$ 'ist ganz wechselnd; wenn iiberhaupt, so ist nur wenig Wasser als Konstitutionswasser aufzufassen.

Harmotom. - $\mathrm{Al}_{6} \mathrm{X}_{6} \mathrm{Ba}_{3} .15 \mathrm{H}_{2} \mathrm{O}$. Das Verhältnis zwischen $\mathrm{Si}_{3} \mathrm{O}_{8}$ und $\mathrm{SiO}_{4}$ ist gewöhnlich gleich $1: 1$; meistens ist etwas Bat durch $\mathrm{H}_{2}$ oder Alkalien ersetzt; man betrachtet Harmotom allgemein als fast analog dem Phillipsit.

Lev ynit. $-\mathrm{Al}_{6} \mathrm{X}_{6} \mathrm{Ca}_{3} .15 \mathrm{H}_{2} \mathrm{O} . \quad \mathrm{SiO}_{4}: \mathrm{Si}_{3} \mathrm{O}_{8}=3: 2$; geringer Ersatz des Calciums durch Alkalien. Nach Damouns Versuchen zu urteilen, scheint etwa $1 / 3$ des Wassers Konstitutionswasser zu sein.

Chabazit. - Derselbe steht zwischen $\mathrm{Al}_{6} \mathrm{X}_{6} \mathrm{Ca}_{3} \cdot 18 \mathrm{H}_{2} \mathrm{O}$ und $\mathrm{Al}_{3} \mathrm{X}_{3} \mathrm{Na}_{3} \cdot 9 \mathrm{H}_{2} \mathrm{O}$ mit der Proportion $\mathrm{SiO}_{4}: \mathrm{Si}_{3} \mathrm{O}_{8}=1: 1$, und zeigt scheinbar das Verhältnis eines Metasilikats. Nach Dowtare liefert er beim Schmelzen Kieselsäure und Anorthit. Im Calciumsalz hat man vier Wassermoleküle und im Natriumsalz zwei Moleküle als Konstitutionswasser zu betrachten, was die Endformeln liefert:

$$
\begin{aligned}
& \mathrm{Al}_{4} \mathrm{X}_{6} \mathrm{Ca}_{3} \mathrm{H}_{4}\left(\mathrm{AlH}_{2} \mathrm{O}_{2}\right)_{2} \cdot 14 \mathrm{H}_{2} \mathrm{O}, \\
& \mathrm{Al}_{2} \mathrm{X}_{3} \mathrm{Na}_{3} \mathrm{H}_{2}\left(\mathrm{AlH}_{2} \mathrm{O}_{2}\right) \cdot 7 \mathrm{H}_{2} \mathrm{O} .
\end{aligned}
$$

Gmelinit. - $\mathrm{Al}_{3} \mathrm{X}_{3} \mathrm{Na}_{3} \cdot 9 \mathrm{H}_{2} \mathrm{O}$, oder wie Chabazit olne Calciumsalz, jedoch mit dem Verhältnis $\mathrm{SiO}_{4}: \mathrm{Si}_{3} \mathrm{O}_{8}=1: 2$; wahrschein.. lich sind zwei Moleküle Wasser Konstitutionswasser.

Hydronephelith. - $\mathrm{Al}_{3}\left(\mathrm{SiO}_{4}\right)_{3} \mathrm{Na}_{2} \mathrm{H} \cdot 3 \mathrm{H}_{2} \mathrm{O}$, offenbar ein Nephelinderivat.

Offretit. - Die Analyse von Gonnard stimmt scharf mit der Formel überein:

$$
2 \mathrm{Al}_{3}\left(\mathrm{Si}_{3} \mathrm{O}_{8}\right)_{3} \mathrm{~K}_{3} \cdot 8 \mathrm{H}_{2} \mathrm{O}+\mathrm{Al}_{3}\left(\mathrm{SiO}_{4}\right)_{3} \mathrm{CaH} .8 \mathrm{H}_{2} \mathrm{O} \text {. }
$$

So viel über die erste Gruppe der Zeolithe, welche durch das vollkommen einheitliche Verhältnis von $\mathrm{Al}: \mathrm{X}=6: 6$ oder $3: 3$ charakterisiert ist, gleichviel ob unter den anderen metallischen Komponenten zwei- oder einwertige Basen vorherrschen. In ähnlicher Weise ist die zweite Gruppe durch das Verhältnis $\mathrm{Al}: \mathrm{X}=4: 6$ 
oder 2:3 definiert, während die hauptsächliche Abwechselung im Wassergehalt besteht; die Glieder dieser Gruppe sind im einzelnen folgende:

Foresit. - $\mathrm{Al}_{4}\left(\mathrm{SiO}_{4}\right)_{6} \mathrm{CaH}_{10} \cdot \mathrm{H}_{2} \mathrm{O}$.

Skolezit. - $\mathrm{Al}_{4}\left(\mathrm{SiO}_{4}\right)_{6} \mathrm{Ca}_{2} \mathrm{H}_{8} \cdot 2 \mathrm{H}_{2} \mathrm{O}$; beim Schmelzen liefert er nach Doflter Anorthit und wird nach Lembera durch fortgesetzte Einwirkung von Sodalösungen in Natrolith verwandelt; bei $300^{\circ}$ verliert er etwa $1 / 3$ seines Wassers, welches er an feuchter Luft wieder aufnimmt.

Laumontit. - $\mathrm{Al}_{4} \mathrm{X}_{6} \mathrm{Ca}_{2} \mathrm{H}_{8} \cdot 4 \mathrm{H}_{2} \mathrm{O} ; \mathrm{SiO}_{4}: \mathrm{Si}_{3} \mathrm{O}_{8}=5: 1$. Er liefert beim Schmelzen Anorthit und ein Pyroxenmineral und verliert bei $300^{\circ}$ etwa die Hälfte seines Wassers.

Heulandit. $-\mathrm{Al}_{4} \mathrm{X}_{6} \mathrm{Ca}_{2} \mathrm{H}_{8} \cdot 6 \mathrm{H}_{2} \mathrm{O}$ mit dem Verhältnis $\mathrm{SiO}_{4}: \mathrm{Si}_{3} \mathrm{O}_{8}=1: 1$; er liefert nach DoELTER beim Schmelzen neben Kieselsäure "Calciumaugit". Diese Beobachtung würde am besten mit der Metasilikatformel $\mathrm{Al}_{2} \mathrm{Ca}\left(\mathrm{SiO}_{3}\right)_{6} \mathrm{H}_{4} \cdot 3 \mathrm{H}_{2} \mathrm{O}$ übereinstimmen; beide Formeln sind empirisch gleich und in Übereinstimmung mit dem Verhalten des Heulandits beim Entwässern.

Brewsterit. - Wie Heulandit, doch mit Baryum und Strontium anstatt Calcium.

Epistilbit. - Isomer mit Heulandit, jedoch scheinbar mit festerer Bindung des Wassers; vielleicht ist $\mathrm{Al}$ teilweise durch $\mathrm{AlH}_{2} \mathrm{O}_{2}+\mathrm{H}_{2}$ ersetzt.

Stilbit. - $\mathrm{Al}_{4} \mathrm{X}_{6} \mathrm{Ca}_{2} \mathrm{H}_{8} \cdot 8 \mathrm{H}_{2} \mathrm{O}$ mit dem Verhältnis $\mathrm{SiO}_{4}: \mathrm{Si}_{3} \mathrm{O}_{8}=1: 1$. Er verhält sich beim Schmelzen wie Heulandit, liefert aber bisweilen Anorthit. Die Abweichungen in der Zusammensetzung sind wahrscheinlich eine Folge des wechselnden Verhältnisses zwischen den Ortho- und Trisilikatgruppen.

Faujasit. - $\mathrm{Al}_{4} \mathrm{X}_{6} \mathrm{Na}_{2} \mathrm{CaH}_{8} \cdot 15 \mathrm{H}_{2} \mathrm{O} ; \mathrm{SiO}_{4}: \mathrm{Si}_{3} \mathrm{O}_{8}=2: 1$. Auch läfst sich Faujasit auffassen als ein Salz der Säure $\mathrm{H}_{6} \mathrm{Si}_{2} \mathrm{O}_{7}$ mit der Formel $\mathrm{Al}_{4}\left(\mathrm{Si}_{2} \mathrm{O}_{7}\right)_{5} \mathrm{CaNa}_{2} \mathrm{H}_{14} \cdot 12 \mathrm{H}_{2} \mathrm{O}$. Da das Mineral bei der Zersetzung mit Salzsäure nicht gelatinös wird, so gehört es vielleicht nicht zu den Orthosilikaten.

Natrolith. - $\mathrm{Al}_{2}\left(\mathrm{SiO}_{4}\right)_{3} \mathrm{Na}_{2} \mathrm{H}_{4}$. Der Charakter seines Wassers ist zweifelhaft; beim Schmelzen liefert er das Mineral Nephelin $\mathrm{Al}_{3}\left(\mathrm{SiO}_{4}\right)_{3} \mathrm{Na}_{3}$, bisweilen mit sehr geringem Ersatz von $\mathrm{SiO}_{4}$ durch $\mathrm{Si}_{3} \mathrm{O}_{8}$.

Die sorgfältige Prüfung der obigen Formeln wird ergeben, dals dieselben, soweit die Angaben zur Zeit reichen, alle zu Anfang dieser Zeilen abgeleiteten Bedingungen erfüllen. Sie stimmen mit den ver- 
öffentlichten Analysen überein, erklären völlig ihre Verschiedenheiten und entsprechen in ihrem 'Iypus den beiden Feldspatmolekülen, deren Symbole angefülırt worden sind. Sie unterscheiden sich hauptsüchlich hinsichtlich ihres Wassergehaltes, und diese Verschiedenheiten sind nunmehr verständlich. Aufserdem liefern mehrere Arten beim Sehmelzen Anorthit, was die mitgeteilten Formeln mit den angenommenen 'Typen und zugleich mit der wahrscheinlichen Entstehung von Zeolithen aus Feldspaten in Zusammenhang bringt. Thre Unterschiede entsprechen beobachteten Unterschieden, und sie weisen auf die Richtung der Untersuchung hin, durch welche sie endgültig eingereiht, oder ausgeschaltet werden können. Leisten die Formeln nicht mehr als uur die Erfüllung der letztgenannten Funktion, so besitzen sie dennoch einen Wert, welcher sie der Betrachtung wert macht. Lassen wir die Unterscheidung zwischen Krystall- und Konstitutionswasser aufser acht und übergehen der Kürze halber die wenigen Baryumstrontiumsalze, so lassen sich die normalen Zeolithe, wenn ich sie so nennen darf, nümlicl,, wie folgt, anordnen :

Erste Gruppe:

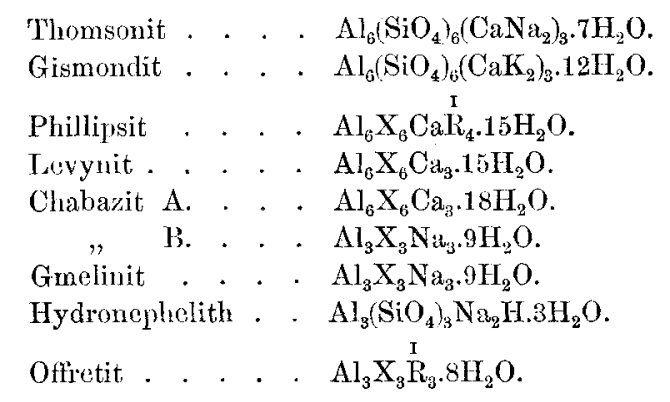

Zweite Gruppe:

\begin{tabular}{|c|c|c|}
\hline & & \\
\hline $\mathrm{K} \theta$ & & $\mathrm{H}_{2}$ \\
\hline 40 & & \\
\hline Ien & & $\mathrm{B}_{2} \mathrm{O}$. \\
\hline & & $\mathrm{I}_{2} \mathrm{O}$. \\
\hline tilbit & & $\mathrm{Al}_{4} \mathrm{X}_{6} \mathrm{Ca}_{2} \mathrm{H}_{8} \cdot 8 \mathrm{H}_{2} \mathrm{O}$ \\
\hline Faujasi & & $\mathrm{Al}_{4} \mathrm{X}_{6} \mathrm{Na}_{2} \mathrm{CaH}_{8} .15 \mathrm{H}_{2}$ \\
\hline & & $\mathrm{Al}_{2}\left(\mathrm{SiO}_{4}\right)_{3} \mathrm{Na}_{2} \mathrm{If}_{4}$ \\
\hline
\end{tabular}

Nunmehr sind noch verschiedene Zeolithe zugleich mit einigen verwandten Mincralien zu betrachten, welche Verbindungen eines anderen Typus als die in obiger Tabelle angeführten zu sein scheinen. 
In einer früheren Abhandlung ${ }^{1}$ habe ich zwei dieser Zeolithe, Ptilolith und Mordenit, schon besprochen; ihnen verwandt scheint ein dritter, der in neuerer Zeit entdeckte Laubanit zu sein. Im Ptilolith und Mordenit liegen Salze der zweibasischen Säure $\mathrm{H}_{2} \mathrm{Si}_{2} \mathrm{O}_{5}$ vor, während Laubanit ein Metasilikat von genau dem gleichen Typus ist. Die beiden erstgenannten Mineralien sind in gleicher Weise Gemenge gewisser fundamentaler Salze, während Laubanit sich anders verhält; ihre Formeln sind folgende:

Ptilolith und Mordenit.

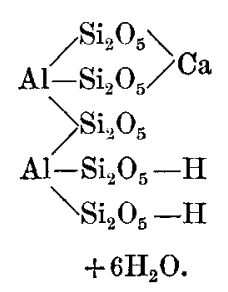

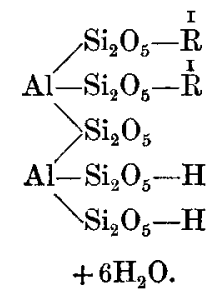

Laubanit.

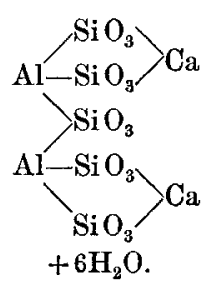

Die Verwandtschaft tritt so deutlich hervor, dafs eine weitere Erklärung unnötig ist. Laubanit lälst sich auch so schreiben, dafs er zur normalen Reihe gehören würde, jedoch ist diese Konstitution sicherlich vorzuziehen.

Ein weiterer Zeolith, Analcim, läfst sich schreiben: $\mathrm{H}_{4} \mathrm{Na}_{2} \mathrm{Al}_{2} \mathrm{X}_{3}$, worin $1 / 6$ des $\mathrm{X}=\mathrm{Si}_{3} \mathrm{O}_{8}$ ist; danach würde das Mineral in die zweite Gruppe der normalen Zeolithe fallen und in nahe Beziehung zum Natrolith kommen. Indes ist der Analcim dem wasserfreien Leucit isomer, und, wie LeMBerg gezeigt hat, lassen sich Analcim und Leucit durch fortgesetzte Einwirkung von Alkalilösungen gegenseitig in einander umwandeln. Demgemäls müssen beide Arten als nahe verwandt und als vom gleichen Typus betrachtet werden; ihre einfachsten Formeln sind:

Leucit.<smiles>[R]O[AsH][O-]</smiles>

Analcim.

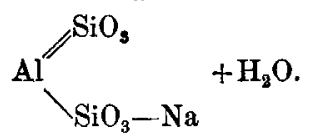

Beide Mineralien kommen indes in Feldspate verwandelt vor, und die Frage ist noch offen, ob ihre Formeln nicht als polymere der oben angegebenen aufzufassen sind, damit man diese Umwandlung verstehen kann. Die gleiche Frage liegt beim Spodumen vor, einer

\footnotetext{
1 Amer. Journ. Se. (August 1892). 
Verbindung von ähnlicher Formel, welche sich leicht in Albit und Eukryptit spaltet. Diese Punkte werde ich an anderer Stelle besprechen.

Ferner liegen noch drei andere Mineralien, welche bisweilen zu den Zeolithen gerechnet werden, aber kein Aluminium enthalten, vor, nämlich der Okenit, Gyrolith und Apophyllit. Diese Mineralien sind unter einander ohne Zweifel verwandt, denn Apophyllit lälst sich künstlich aus Okenit darstellen, und Gyrolith kommt in der Natur sowohl als Muttersubstanz wie als sekundäres Produkt des Apophyllits vor. In New-Almaden ist Apophyllit das sekundäre Mineral, während der Gyrolith von Nova Scotia durch Umwandlung des ersteren entstanden zu sein scheint. Nimmt man für alle drei Mineralien analoge Struktur an, so lassen sie sich sehr einfach als Salze der Säure $\mathrm{H}_{6} \mathrm{Si}_{2} \mathrm{O}_{7}$ darstellen, unter welcher Voraussetzung ihnen folgende Formeln zukommen:
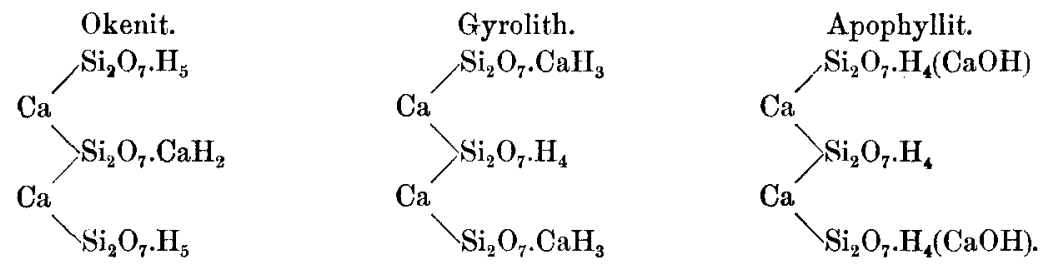

Das einwertige $\mathrm{CaOH}$ im Apophyllit ist teilweise durch Kalium und das Hydroxyl häufig durch Fluor ersetzt. Als ein Glied der gleichen Gruppe mit der Formel $\mathrm{Ca}_{2}\left(\mathrm{Si}_{2} \mathrm{O}_{7}\right)_{3} \mathrm{Ca}_{3} \mathrm{H}_{8} \cdot 9 \mathrm{H}_{2} \mathrm{O}$ lärst sich ferner der noch etwas unsichere Plombièrit auffassen.

$\mathrm{Da}$ ich in dieser Abhandlung lediglich eine vorläufige Darstellung der Ansichten, zu welchen ich gelangt bin, geben wollte, so hielt ich es für überflüssig, die Seiten mit Litteraturhinweisen und mit Besprechungen von Analysen zu füllen. Ich habe eine grolse Anzahl von Angaben geprüft, und es ist meine Absicht, den Gegenstand in nächster Zeit ausführlich durchzuarbeiten, bei welcher Gelegenheit ich auch Beweismaterial in genügender Fülle bringen werde.

U. S. Geol. Survey, Washington, den 20. Juni 1894.

Bei der Redaktion eingegangen am 26. Juli 1894. 